\title{
A Novel Bridging Strategy for Patients Undergoing Emergent Non-Cardiac Surgery with a Recent Coronary Stent
}

Cierra Bruck · Omar Jafar · Jayne Prats · Deepak Bhatt •

Zubair Jafar

Received: May 26, 2018 / Published online: July 11, 2018

(C) The Author(s) 2018

\section{ABSTRACT}

Introduction: Not infrequently, patients undergoing dual antiplatelet therapy for a recent cardiac stent develop a need for a noncardiac surgery. Most of these surgeries can be delayed while the antiplatelet treatment is stopped and normal platelet function returns in order to avoid potential surgical complications and excessive bleeding. However, there are a number of patients who require urgent surgery where the procedure cannot be postponed. To date, no agents have been proven to bridge the patient off dual-antiplatelet therapy.

Methods: A 46-year-old man was admitted to the hospital with an acute anterior wall

The original version of this article was revised: In the original publication, the abstract text, informed consent and disclosure text have been published incorrectly. Now they have been corrected in the original article.

Enhanced Digital Features To view enhanced digital features for this article go to https://doi.org/10.6084/ m9.figshare.6635303.

C. Bruck · O. Jafar · Z. Jafar $(\bowtie)$

The Heart Center-Vassar Brothers Medical Center, Poughkeepsie, NY, USA

e-mail: mjafar@health-quest.org

J. Prats

Elysis, LLC, Carlisle, MA, USA

D. Bhatt

Brigham and Women's Heart and Vascular Center, Boston, MA, USA myocardial infarction. He was urgently taken to the catheterization lab where he was found to have a totally occluded proximal left anterior descending coronary artery (LAD). He was successfully stented with a drug-eluting stent of the LAD, but subsequently developed a sarcoma 2 months later that required urgent surgery. The novel direct-acting, reversible $\mathrm{P}_{2} \mathrm{Y}_{12}$ receptor inhibitor cangrelor was used to bridge this patient followed by ongoing antiplatelet treatment, allowing surgery for the removal of a soft tissue sarcoma.

Results: Cangrelor was successfully used to bridge a patient with a recent stent placement and current antiplatelet treatment undergoing the removal of a soft tissue sarcoma.

Conclusion: This case report demonstrates the use of a novel, now currently available, shortacting antiplatelet agent that can be used for bridging patients undergoing non-cardiac surgery who had a recent myocardial infarction and stent placement.

Keywords: Antiplatelet therapy; Bridging strategies; Non-cardiac surgery; Stent thrombosis

\section{INTRODUCTION}

Not infrequently, patients undergoing dual antiplatelet therapy for a recent cardiac stent subsequently require non-cardiac surgery. These 
surgeries can mostly be delayed while the antiplatelet treatment is stopped and normal platelet function returns in order to avoid potential surgical complications and excessive bleeding. However, for a number of patients surgery is urgent and the procedure cannot be delayed. This situation is challenging as the risks of bleeding due to the antiplatelet therapy and the thrombotic risks of stopping the antiplatelet must be weighed and compared before the surgery [4]. At the present time, there are no specific guidelines on how to treat a patient on antiplatelet therapy who requires urgent noncardiac surgery. This lack of information is due to the absence of randomized trials exploring this issue. However, a few studies have tested different types of bridging strategies. Bridging in this context refers to "the temporary administration of an antithrombotic agent preoperatively in order to minimize the time period that a patient remains free from antithrombotic protection between withdrawal of oral antiplatelet therapy and the time of surgery" (Anastasiou et al., p 137 [1]). The different agents utilized in these bridging strategies include heparin and glycoprotein (GP) IIb/IIIa inhibitors, both of which have drawbacks, particularly in this setting. However, cangrelor, a novel direct-acting, reversible $\mathrm{P}_{2} \mathrm{Y}_{12}$ receptor inhibitor can be used similarly to adenosine diphosphate (ADP) receptor antagonists. Cangrelor can be administered intravenously, and it has a rapid onset and offset, making it valuable for use in urgent peri-operative situations where there is a high risk of stent thrombosis. In the specific case reported here, cangrelor was used in an innovative method of bridging a high-risk patient with a recent stent placement who required emergent surgery to remove a soft tissue sarcoma in his left chest region.

\section{CASE INFORMATION}

A 46-year-old man was admitted to the hospital with an acute anterior wall myocardial infarction. He was urgently taken to the catheterization lab where he was found to have a totally occluded proximal left anterior descending coronary artery (LAD). The LAD was successfully stented, following which the patient had a $40 \%$ mid-right coronary artery lesion and ejection fraction of approximately $40-45 \%$ with an apical wall motion abnormality, which was subsequently confirmed on an echocardiogram. He did well post intervention without any further chest pain, dyspnea, or symptoms consistent with myocardial ischemia. He had an unremarkable post-procedural course and was discharged on combined therapy of aspirin $325 \mathrm{mg}$ a day, atorvastatin $80 \mathrm{mg}$ a day, metoprolol $50 \mathrm{mg}$ twice daily, prasugrel $10 \mathrm{mg}$ a day, and ramipril $5 \mathrm{mg}$.

Two months after this successful cardiac procedure, the patient returned with a new chest mass. He now presented for preoperative clearance for a possible sarcoma of the left chest. The ejection fraction at the patient's first presentation was $40 \%$, and a subsequent repeat echocardiogram showed well-preserved left ventricular systolic function of 55\%.

Due to his recent anterior wall myocardial infarction and placement of drug-eluting stent, the patient was currently taking prasugrel and aspirin. Surgeons in the General Surgery department were consulted, and they were not willing to operate on the patient while he was on aspirin and prasugrel given the invasive nature of the procedure and the high risk of bleeding. It was then decided to use cangrelor as a bridging agent for this procedure.

The patient discontinued prasugrel 2 days prior to being admitted to the hospital, but continued on aspirin. He was then started on $4 \mathrm{mcg} / \mathrm{kg} / \mathrm{min}$ of cangrelor and continued this treatment for the next 3 days. The drug was stopped $1 \mathrm{~h}$ prior to entrance into the operating room where he underwent a biopsy and excision of the soft tissue sarcoma. The patient tolerated the procedure well. He was then started on $60 \mathrm{mg}$ of prasugrel $48 \mathrm{~h}$ after the procedure and continued on this agent at a dose of $10 \mathrm{mg}$ per day. He experienced no further issues. All of his post-operative checkups and tests were normal, and the patient experienced no complications.

Informed consent was obtained from the individual participant for inclusion in the study. 


\section{DISCUSSION}

The patient presented with a soft tissue sarcoma that needed urgent excision. This proved a challenge as the patient was currently on aspirin and prasugrel therapy, and withdrawal of the ADP receptor antagonist $\left(\mathrm{P}_{2} \mathrm{Y}_{12}\right)$ would put the patient at high risk of acute stent thrombosis. To avoid this risk, antiplatelet therapy needed to continue for as close to the surgical procedure as possible. There have been several small studies using either heparin or GP IIb/IIIa receptor inhibitors as bridging agents; however, the results gave no clear indication of whether these agents were successful. The direct, fast-acting and reversible nature of cangrelor makes it an appealing option for avoiding thrombotic risks as well as the risk of surgical bleeding.

The pharmacologic profiles of such oral antiplatelets as clopidogrel, prasugrel, and ticagrelor have led researchers to study different agents that could potentially be used in antiplatelet-bridging strategies. Clopidogrel and prasugrel are prodrugs whose active metabolites bind to the $\mathrm{P}_{2} \mathrm{Y}_{12}$ receptor for the lifespan of the platelet (about 7-10 days), thus requiring drug discontinuation 5-7 days before surgery [3]. Although $\mathrm{P}_{2} \mathrm{Y}_{12}$-receptor binding by ticagrelor is reversible, discontinuation 5 days before surgery is still recommended. Such waiting periods are unfavorable to the patient as he/she is without an antithrombotic medication for 5-7 days, which puts that patient at risk for acute stent thrombosis.

Given our patient's recent history, we were concerned that heparin or GP IIb/IIIa receptor inhibitors would cause significant complications. Unfractionated heparin and low molecular weight heparins are potential agents for possible bridging strategies. Heparin can affect platelet reactivity, either positively or negatively, making its effect inconsistent [1]. Two studies have evaluated the perioperative use of heparins in patients with coronary stents; neither revealed any consistent protective effect of these agents against stent thrombosis. These studies also found an increase in bleeding events [1]. The lack of evidence supporting the safety of heparins discourages their use for bridging situations. Intravenous GP IIb/IIIa inhibitors have also been researched as potential bridging agents. The two small-molecule GP IIb/IIIa inhibitors with reversible inhibition are eptifibatide and tirofiban [1], although their offset of effect is several hours. Despite their theoretically applicable pharmacologic profile, evidence from randomized trials is lacking, and the results of small nonrandomized or retrospective studies have been inconsistent [1]. The lack of consistent success with these bridging agents led us to consider cangrelor.

Cangrelor is an intravenous P2Y12 inhibitor with a very short plasma half-life of about 3-5 min that can reversibly block the platelet P2Y12 receptor. This short half-life and direct administration allows for a rapid offset of action within $1 \mathrm{~h}$ of cessation of administration, while the onset of action is immediate [1]. Cangrelor's ability to work directly on the P2Y12 receptor on platelets-as compared with GP IIb/IIIa inhibitors-potentially makes it a valuable bridging medication. In the BRIDGE study, cangrelor for bridging thienopyridine-treated patients to CABG surgery was tested against a placebo. The results showed that cangrelor maintained an adequate level of platelet inhibition that was similar to the level expected with clopidogrel [2].

\section{CONCLUSION}

Cangrelor was successfully used to bridge a patient with a recent stent placement and currently on antiplatelet treatment who required surgery for the removal of a soft tissue sarcoma. It is not uncommon for some patients on antiplatelet therapy to subsequently require urgent non-cardiac surgery. There is currently a lack of clinical trials and no specific guidelines on how to treat a patient on an oral ADP receptor antagonist who requires urgent non-cardiac surgery. Some trials have evaluated the bridging ability of agents such as heparin and GP IIb/IIIa inhibitors, but the results have been inconsistent and unsuccessful. The pharmacologic characteristics of the P2Y12 receptor inhibitor cangrelor, namely, its fast onset and offset of effect, potentially make it an ideal bridging 
agent. This case report demonstrates the use of this novel, now currently available, short-acting antiplatelet agent that can be used for bridging patients undergoing non-cardiac surgery who had a recent myocardial infarction and stent placement.

\section{ACKNOWLEDGEMENTS}

We thank the participants of the study.

Funding. No funding or sponsorship was received for this study or publication of this article. The article processing charges were funded by the authors.

Editorial Assistance. We thank Dr. Dominick Angiolillo and Dr. Michael Firstenberg for their editorial assistance during the writing of this case study.

Authorship. All named authors meet the International Committee of Medical Journal Editors (ICMJE) criteria for authorship for this article, take responsibility for the integrity of the work as a whole, and have given their approval for this version to be published.

Disclosures. Dr. Jayne Prats is a former employee of The Medicines Company, previous sponsor of cangrelor; consultant to Chiesi-USA, who currently markets cangrelor. Ms. Cierra Bruck, Mr. Omar Jafar and Dr. Zubair Jafar all have nothing to disclose. Dr. Deepak Bhatt discloses the following relationships - Advisory Board: Cardax, Elsevier Practice Update Cardiology, Medscape Cardiology, Regado Biosciences; Board of Directors: Boston VA Research Institute, Society of Cardiovascular Patient Care, TobeSoft; Chair: American Heart Association Quality Oversight Committee; Data Monitoring Committees: Baim Institute for Clinical Research (formerly Harvard Clinical Research Institute, for the PORTICO trial, funded by St. Jude Medical, now Abbott), Cleveland Clinic, Duke Clinical Research Institute, Mayo Clinic, Mount Sinai School of Medicine, Population Health Research Institute; Honoraria: American College of Cardiology (Senior Associate Editor, Clinical Trials and News,
ACC.org; Vice-Chair, ACC Accreditation Committee), Baim Institute for Clinical Research (formerly Harvard Clinical Research Institute; REDUAL PCI clinical trial steering committee funded by Boehringer Ingelheim), Belvoir Publications (Editor in Chief, Harvard Heart Letter), Duke Clinical Research Institute (clinical trial steering committees), HMP Global (Editor in Chief, Journal of Invasive Cardiology), Journal of the American College of Cardiology (Guest Editor; Associate Editor), Population Health Research Institute (for the COMPASS operations committee, publications committee, steering committee, and USA national co-leader, funded by Bayer), Slack Publications (Chief Medical Editor, Cardiology Today's Intervention), Society of Cardiovascular Patient Care (Secretary/Treasurer), WebMD (CME steering committees); Other: Clinical Cardiology (Deputy Editor), NCDRACTION Registry Steering Committee (Chair), VA CART Research and Publications Committee (Chair); Research Funding: Abbott, Amarin, Amgen, AstraZeneca, Bayer, Boehringer Ingelheim, Bristol-Myers Squibb, Chiesi (including for his role as co-Chair of CHAMPION PHOENIX), Eisai, Ethicon, Forest Laboratories, Idorsia, Ironwood, Ischemix, Lilly, Medtronic, PhaseBio, Pfizer, Regeneron, Roche, Sanofi Aventis, Synaptic, The Medicines Company (including for his role as co-Chair of the CHAMPION trials); Royalties: Elsevier (Editor, Cardiovascular Intervention: A Companion to Braunwald's Heart Disease); Site Co-Investigator: Biotronik, Boston Scientific, St. Jude Medical (now Abbott), Svelte; Trustee: American College of Cardiology; Unfunded Research: FlowCo, Merck, PLx Pharma, Takeda.

Compliance with Ethics Guidelines. Informed consent was obtained from all individual participants for being included in the study.

Data Availability. Data sharing is not applicable to this article as no datasets were generated or analyzed during the current study.

Open Access. This article is distributed under the terms of the Creative Commons Attribution-NonCommercial 4.0 International License (http://creativecommons.org/licenses/ by-nc/4.0/), which permits any 
noncommercial use, distribution, and reproduction in any medium, provided you give appropriate credit to the original author(s) and the source, provide a link to the Creative Commons license, and indicate if changes were made.

\section{REFERENCES}

1. Anastasiou I, Petousis S, Hamilos M. Current strategies for bridging dual antiplatelet therapy in patients requiring surgery. Interv Cardiol. 2015;7(2):137-48.
2. Angiolillo DJ, Firstenberg MS. Bridging antiplatelet therapy with cangrelor in patients undergoing cardiac surgery. JAMA. 2012;307(3):265-74.

3. Keating GM. Cangrelor: a review in percutaneous coronary intervention. Drugs. 2015;75(12):1425-34.

4. Savonitto S, D'Urbano M, Caracciolo M, et al. Urgent surgery in patients with a recently implanted coronary drug-eluting stent: a phase II study of 'bridging' antiplatelet therapy with tirofiban during temporary withdrawal of clopidogrel. $\mathrm{Br} \mathrm{J}$ Anaesth. 2010;104(3):285-91. 